\section{A REFRACTIVE COLLIMATOR FOR SYNCHROTRON RADIATION}

A lens will collimate the radiation from a point source placed in its focus. Thus the development of compound refractive lenses for focusing X-rays [1] has the corollary that such lenses can be used for collimation. While the present third generation synchrotron radiation facilities provide beams from insertion devices that are relatively well-collimated, the high brilliance of these sources means that many high resolution experiments are now being done that would benefit from an even more collimated X-ray beam. In this context, one considers high-energy resolution for, e.g., inelastic or nuclear scattering measurements, as well as high-angular resolution (or, in fact, nearly any measurement making use of Bragg reflections in perfect crystals - including polarization control and analysis). Here we show that a refractive lens may be used to collimate the radiation from an undulator to $<3 \mu \mathrm{rad}$ full width at half maximum (FWHM), with only a small $(\sim 10 \%)$ loss in intensity [2].

The essential concept of our collimator is shown in Figure 1. A piece of material with holes drilled normal to the beam propagation direction acts as an X-ray lens [1]. Collimation is achieved by choosing the number of holes so that the distance to the source point is the focal length of the lens. In selecting the material, it is important to avoid photoelectric absorption. Therefore, for low- to

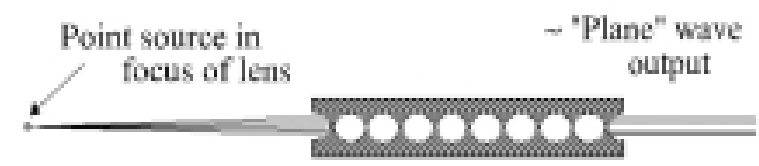

Compound Refractive Lens (CRL) $-45 \mathrm{~m}$ Focal length

Fig.1: Principle behind refractive collimator. Matching the focal length to the distance to the point source leads to collimation of transmitted radiation. medium-energy X-rays (approximately 10 to 40 $\mathrm{keV}$ ), one should choose materials with a low atomic number (at higher energies, Compton scattering must be considered). After extremely promising results using plastic lenses [3], we had some lenses fabricated out of beryllium. While a variety of practical concerns (lens acceptance, transmission, aberrations, etc.) are relevant to lens fabrication, here we do not go into detail but refer the reader to longer papers (e.g. [4]). We do note, however, that the long focal length for collimation makes these lenses relatively efficient, as it has large apertures without too much absorption.

We tested the collimator at the beamline BL47XU [5]. $18.5 \mathrm{keV} \mathrm{X}$-rays were selected from the third harmonic of an in-vacuum undulator using a cryogenically-cooled Si monochromator [6]. In order to measure the divergence of the incident beam and the effect of the collimator on that divergence, we used a pair of Si (555) reflections in a dispersive $(+,+)$ setting. In this geometry, assuming the bandwidth of the incident beam is sufficiently large, scanning the second crystal provides a map of the divergence incident on the first crystal with a 2:1 resolution: if the width of the rocking curve of the second crystal is $\Delta \theta$, the divergence of the beam incident on the pair is $\Delta \theta / 2$. The effect of the beryllium refractive collimator $(\phi$ $2.2 \mathrm{~mm}$ holes, 13 interfaces of 100 microns each) is shown in Figure 2. It reduced the beam divergence from 11.5 to $<3 \mu \mathrm{rad}$ (FWHM) an improvement of about a factor of four, while preserving $89 \%$ of the incident flux. Similar results were obtained using a lens of seven interfaces at $14.4 \mathrm{keV}$, with the beam collimation improving from 14 to $<3 \mu \mathrm{rad}$ (FWHM) and transmission of about $92 \%$.

We have shown that a refractive collimator is an efficient method for collimating an undulator beam, leading to a significantly reduced divergence without a large sacrifice in intensity. Compared with crystal optics, our method has the very important advantage that it does not lead to an increased beam size. In addition, the bandwidth of the collimator $(>\sim 1 \%)$ is much larger than an asymmetric reflection, and this collimator neither displaces nor modifies the direction of transport of the X-ray beam. Also, the strong chromatic 
aberration of asymmetric crystals (leading to an energy-angle correlation in the output beam) does not appear.

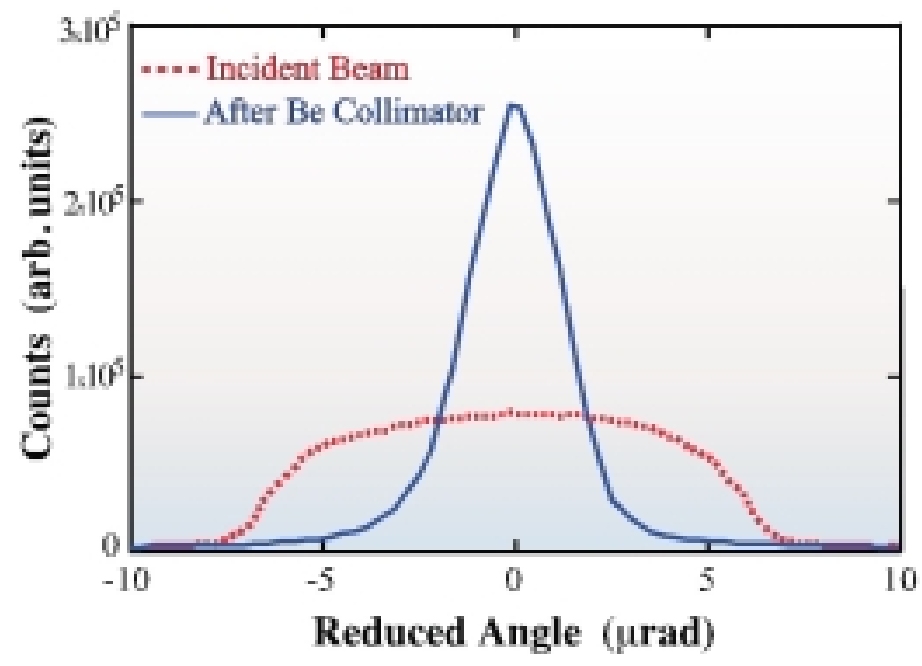

Fig.2: Effect of beryllium collimator at $18.5 \mathrm{keV}$. Dashed line shows beam divergence without the collimator. Solid line shows divergence measured after the collimator. X-ray divergence is reduced from 11.5 to < $3.0 \mu$ rad (FWHM) while $89 \%$ of incident intensity is transmitted. Note that "reduced" angle for $x$-axis corresponds to measured divergence of $X$-ray beam (see text).

Alfred Q.R. Baron

SPring-8 / JASRI

E-mail: baron@spring8.or.jp

\section{References}

[1] A. Snigirev et al., Nature 384 (1996) 49 .

[2] A.Q.R. Baron et al., to be published in $J$. Synchrotron Rad..

[3] A.Q.R. Baron et al., Appl. Phys. Lett. 74 (1999) 1492.

[4] P. Ellaume, Nucl. Instrumen. and Meth. A $\mathbf{4 1 2}$ (1998) 483.

[5] Y. Kohmura et al., SPring-8 Information (in Japanese) Vol. 3, No. 4 (1998) 28.

[6] T. Mochizuki et al., work in progress.

\section{ATOMIC IMAGING AROUND Zn IN GaAs:Zn USING MULTIPLE -ENERGY X-RAY HOLOGRAPHY}

The X-ray fluorescence holography makes it possible to obtain direct three-dimensional atomic images around atoms emitting fluorescent $\mathrm{X}$-rays [1]. However, only the holograms of single crystals, whose atomic configurations are already known by the X-ray diffraction method, have been measured because of the weakness of the primary X-rays. Therefore, we applied this method to imaging the atomic structure around zinc ( $\mathrm{Zn}$ ) atoms doped in gallium arsenide (GaAs) with synchrotron radiation [2]. X-ray fluorescence holography has two types of experimental techniques. One is called "X-ray fluorescence holography" (XFH) and the other is called "multiple-energy X-ray holography" [3] $(M E X H)$. MEXH is the result of the optical reciprocity theorem applied to $\mathrm{XFH}$ and has the advantage of suppressing the twin image effect. We adopted here the MEXH mode for obtaining the hologram. The incident X-ray energy $9.8 \mathrm{keV}$ was selected, which is between the $\mathrm{Zn}$ and $\mathrm{Ga} \mathrm{K}$ absorption edges, so as to avoid excitation of the $\mathrm{Ga}$ and As X-ray fluorescence. The sample was mounted on a two-axis rotatable stage. Figure 1 shows the experimental setup. The $\mathrm{Zn} \mathrm{K \alpha}$ X-ray fluorescence intensity was measured as a function of azimuthal $(\phi)$ and incident $(\theta)$ angles by an $\mathrm{Si}$ PIN detector, and the resulting holographic pattern was obtained, as shown in Figure 2.

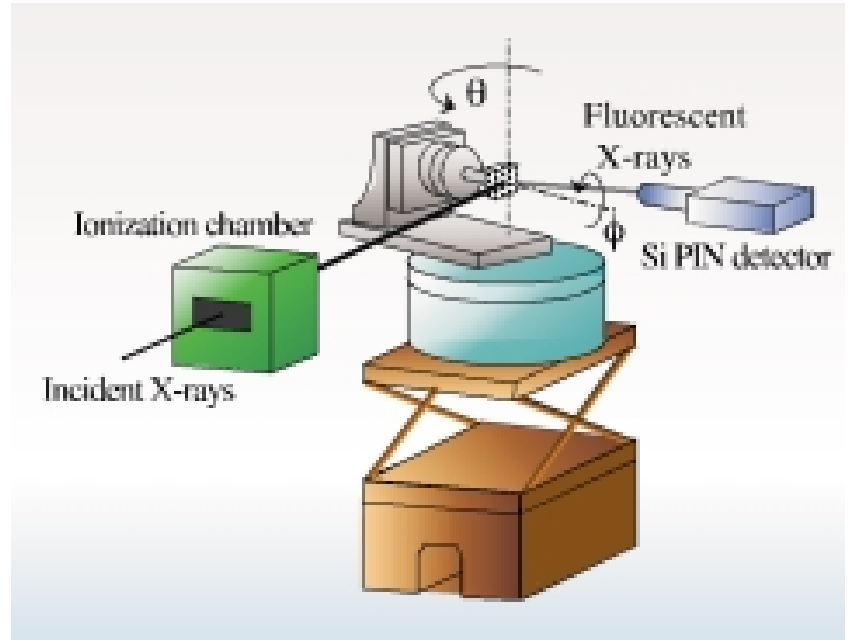

Fig. 1: Experimental setup. 